\title{
Liquid biopsy to identify biomarkers for immunotherapy in hepatocellular carcinoma
}

\author{
Huang $\mathrm{Ao}^{1+}$, Zhang $\mathrm{Xin}^{1 \dagger}$ and Zhou Jian ${ }^{1,2,3,4^{*}}$
}

\begin{abstract}
The past years have witnessed the vigorous development of immunotherapy, mainly immune checkpoint inhibitors (ICls) targeting the programmed cell death-1 (PD-1) protein and its ligand, PD-L1, and cytotoxic T-lymphocyteassociated antigen-4 (CTLA-4). Indeed, ICls have largely revolutionized the management and improved the prognosis of patients with intermediate and advanced hepatocellular carcinoma (HCC). However, biomarker-based stratification of HCC patients for optimal response to $\mathrm{ICI}$ treatment is still of unmet need and again, there exists the necessity to dynamically monitor treatment effect in real-time manner. The role of conventional biomarkers in immunotherapy surveillance is largely limited by spatial and temporal tumor heterogeneity whereas liquid biopsy seems to be promising to circumvent tumor heterogeneity to identify candidate patients who may response to immunotherapy, to dynamically monitor treatment effect and to unveil resistance mechanism. Herein, we provide a thorough review about the potential utility of liquid biopsy in immunotherapy for HCC and discuss its future perspectives.
\end{abstract}

Keywords: Liquid biopsy, Immunotherapy, Immune checkpoint inhibitors, Hepatocellular carcinoma, ctDNA, CTC

\section{Background}

During the past three years, the management of hepatocellular carcinoma (HCC) has been drastically improved with the application of immunotherapy, especially after the advent of immune checkpoint inhibitors (ICIs), namely antibodies targeting the programmed cell death receptor-1 (PD-1), the anti-programmed death 1 ligand (PD-L1), the cytotoxic T-lymphocyte antigen 4 (antiCTLA-4), and most recently, the LAG-3 (Lymphocyteactivation-gene-3). To date, several ICIs and the

\footnotetext{
*Correspondence: zhou.jian@zs-hospital.sh.cn

${ }^{\dagger}$ Huang Ao and Zhang Xin contributed equally to this work.

'Department of Liver Surgery and Transplantation, Zhongshan Hospital, Fudan University; Key Laboratory of Carcinogenesis and Cancer Invasion (Fudan University), Ministry of Education; Shanghai Key Laboratory of Organ Transplantation, Zhongshan Hospital, Fudan University, Shanghai 200032, China

${ }^{2}$ Institute of Biomedical Sciences, Fudan University, Shanghai 200032, China Full list of author information is available at the end of the article
}

combination of ICIs, or ICIs with anti-angiogenic antibody have been approved for the treatment of HCC, either as first-line or second-line agents [1,2]. Although phase III trials of ICI monotherapies in HCC have been negative, such failure has merely influenced the use of ICIs in clinical practice and trials evaluating the combination therapies of ICIs, ICIs with molecular targeted agents or other modalities are still worth expectation [3]. Moreover, evaluation of ICIs as neoadjuvant treatment may provide novel insights and decrease tumor recurrence and metastasis after hepatectomy (Table 1).

Despite that ICIs have resulted in significant therapeutic benefit and prolongation of survival in the whole, the extent of benefit in ICI is not uniform. Not all patients benefit from immunotherapy and most patients would eventually experience disease progression. Although immunotherapies are largely thought to have fewer adverse effects than chemotherapy and molecular

(C) The Author(s). 2021 Open Access This article is licensed under a Creative Commons Attribution 4.0 International License, which permits use, sharing, adaptation, distribution and reproduction in any medium or format, as long as you give appropriate credit to the original author(s) and the source, provide a link to the Creative Commons licence, and indicate if changes were made. The images or other third party material in this article are included in the article's Creative Commons licence, unless indicated otherwise in a credit line to the material. If material is not included in the article's Creative Commons licence and your intended use is not permitted by statutory regulation or exceeds the permitted use, you will need to obtain permission directly from the copyright holder. To view a copy of this licence, visit http://creativecommons.org/licenses/by/4.0/. The Creative Commons Public Domain Dedication waiver (http://creativecommons.org/publicdomain/zero/1.0/) applies to the data made available in this article, unless otherwise stated in a credit line to the data. 
Table 1 Ongoing clinical trials evaluating neoadjuvant immunotherapy in HCC

\begin{tabular}{|c|c|c|c|c|}
\hline Identifier No. & Interventions & Setting & Candidates & Primary outcome \\
\hline NCT04930315 & Camrelizumab + apatinib & $\begin{array}{l}\text { Phase 2, open } \\
\text { label }\end{array}$ & $\begin{array}{l}\mathrm{BCLC} B / \mathrm{C} \text {, or CNLC Ila-IIIb; technic- } \\
\text { ally resectable }\end{array}$ & 1-year tumor recurrence-free rate \\
\hline NCT03630640 & Nivolumab & $\begin{array}{l}\text { Phase 2, open } \\
\text { label }\end{array}$ & $\mathrm{BCLC} A$; receiving electroporation & 2-year RFS \\
\hline $\begin{array}{l}\text { NCT04727307 (AB- } \\
\text { LATE02) }\end{array}$ & Atezolizumab & $\begin{array}{l}\text { Phase 2, open } \\
\text { label }\end{array}$ & Percutaneous Radiofrequency & 2-year RFS \\
\hline NCT04615143 & Atezolizumab; & $\begin{array}{l}\text { Phase 2, single } \\
\text { arm }\end{array}$ & Resectable recurrent $\mathrm{HCC}$ & Pathological response rate \\
\hline NCT04658147 & $\begin{array}{l}\text { Nivolumab With or Without } \\
\text { Relatlimab }\end{array}$ & Phase 1 & Potentially Resectable HCC & $\begin{array}{l}\text { No. of patients who complete treatment } \\
\text { and surgery }\end{array}$ \\
\hline NCT03916627 & Cemiplimab & Phase 2 & Resectable HCC & Significant tumor necrosis \\
\hline
\end{tabular}

targeted therapy, the immune-related adverse events (irAEs) such as myocarditis and thyroiditis could be lethal and it's thus crucial to identify patients who are at risk of therapy related toxicity. Moreover, hyperprogressive disease (HPD) after treatment with ICIs could make the condition much worse. Thus, predictive biomarkers of ICI response are urgently needed to guide treatment decision and patient selection, as well as to better understand and overcome mechanisms of resistance.

\section{Conventional biomarkers for immunotherapy in HCC}

Till now, conventional biomarkers for cancer immunotherapy fall into the following categories: the expression of PD-L1, specific genetic signatures/somatic mutations, tumor mutation burden (TMB), dMMR/MSI (deficient in mismatch repair /microsatellite instability), tumor microenvironment (TME), and gut microbiome. Intuitively, PD-L1 is the ideal biomarker for cancer immunotherapy. Gao et al. reported for the first time PD-L1 expression status in $\mathrm{HCC}$ and provided the rationale of immunotherapy targeting the PD-1/PD-L1 pathway [4]. Other researchers also reported a positive correlation between PD-L1 expression and poor survival in HCC, which further support the usage of ICIs in HCC patients falling within this category [5-7]. Moreover, high expression of $\mathrm{PD}-\mathrm{L} 1$ in the adjacent liver tissue predicted shorter overall survival (OS) and disease-free survival (DFS), meaning the expression of PD-L1 in the peritumoral liver tissue could also be an indicator for adjuvant immunotherapy after radical hepatectomy [8]. However, the approval of nivolumab from CHECKMATE-040 and pembrolizumab from KEYNOTE-224 in the second-line setting for advanced HCC were independent of PD-L1 or immune cell expression $[9,10]$. Moreover, the expression of PD-L1 in HCC was not all consistently high [11], limiting its practical utility as biomarker for immunotherapy in HCC.

Interestingly, for HCC patients who receive liver transplantation (LT) and experience recurrence, expression of PD-L1 is a solid contraindication for ICI treatment [12].
Recurrence of HCC after LT are largely caused by the immunosuppressive microenvironment brought by immunosuppressants and ICI might thus provide survival benefit in these patients. Although cases have been reported that patients with recurrent, disseminated HCC after LT were successfully treated with ICIs $[13,14]$, one should note that introduce of ICIs in these LT recipients inevitably improves immunity and may result in organ rejection and liver failure. Thus, only patients with negative PD-L1 staining should be prescribed ICI to treat tumor recurrence following LT.

TMB has been found highly correlated with the activity of anti-PD-1 therapies across multiple cancers [15] and it has been approved by the United States Food and Drug Administration (FDA) as a criterion for pembrolizumab treatment across different tumor types. In resectable lung cancer, TMB was predictive of the pathological response in patients with neoadjuvant PD-1 blockade [16]. Next generation sequencing (NGS) based genomic profiling work has reported several high TMB associated genetic signatures which may probably promote antitumor immunity and serve as biomarker for ICIs in HCC [17].

Partially like that with TMB, the cancer genome with dMMR contains exceptionally high numbers of mutationassociated neoantigens and may might be recognized by the immune system. For instance, colorectal cancers (CRC) with dMMR had high immunogenicity and were sensitive to immune checkpoint blockade with PD-1 antibodies $[18,19]$ whereas misdiagnosis of MSI or dMMR status in metastatic CRC displaying MSI or dMMR would lead to primary resistance ICIs [20]. In patients with resectable primary gastric cancer, MSI-H could robustly predict immunotherapy response and correlate with longer DFS and OS [21]. Similarly, a recent meta-analysis of randomized controlled trials including the phase III KEYNOTE-062, CheckMate-649, JAVELIN Gastric 100 and KEYNOTE-061 trials, also suggested MSI-H as a positive predictive factor to immunotherapy in patients with advanced gastric cancer [22]. In critically ill patients with end-stage cancers, MSI-H status was proposed to be used in clinical practice as a tumor-agnostic predictive 
biomarker to select candidate for salvage immunotherapy [23]. Indeed, the importance of dMMR/MSI-H status has been further validated in multiple solid tumors and proved to be highly responsive to immunotherapy across different tumors of origin and FDA had already granted an accelerated approval to the anti PD-1 antibody pembrolizumab for adult and pediatric patients with agnostic unresectable/metastatic dMMR or MSI-H cancers [24].

Literally, TME and gut microbiome would surely play an important role in immunotherapy, especially for HCC. Through transcriptome-based immunogram analysis, immunological microenvironment including Wnt/ $\beta$-catenin activation, high combined positive score of PD-L1, and increased infiltration of CD8+ cells in HCC were found significantly associated with longer survival in HCC patients receiving ICI treatments [25]. By analyzing the expression profile of immune-modulating genes in HCC, Cao et al. found 4 immune subtypes of $\mathrm{HCCs}$ with different survivals: patients with $\mathrm{B} 7-\mathrm{H} 3{ }^{\text {low }} /$ $\mathrm{CD} 8^{\text {high }}$ or $\mathrm{CD} 47^{\text {low }} / \mathrm{CD} 8^{\text {high }}$ had the best while those with $\mathrm{B} 7-\mathrm{H} 3^{\text {high }} / \mathrm{CD} 8^{\text {low }}$ or $\mathrm{CD} 47^{\text {high }} / \mathrm{CD} 8^{\text {low }}$ have the worst survival. Such immune classification system may facilitate select HCC patient who would benefit from ICI treatment [26]. HCCs with a discrete population of PD1high CD8+ $\mathrm{T}$ cells might benefit from combined immune checkpoint blockade-based therapies [27]. NASH (non-alcoholic steatohepatitis) -related aberrant $\mathrm{T}$ cell activation causing tissue damage which leads to impaired immune surveillance may be the reason that non-viral HCC, particularly NASH-HCC, might be less responsive to immunotherapy [28].

Gut microbiome, which is critical for the development and regulation of innate and adaptive immunity, may also influence treatment outcomes of ICIs [29, 30]. The gut-liver axis suggests a close connection between liver and gut, whit increasing evidence has proposed a key role of microbiome in the development of HCC [31-34]. Gut microbiome may thus be particularly meaningful in the immunotherapy for HCC. In fact, significant differences on the diversity and composition of gut microbiota in HCC who received ICIs have been found between responders and non-responders [35]. Dynamic variation of the gut microbiome characteristics may be predictive of the efficacy of immunotherapy, contributing to disease monitoring and decision making [36]. Though no association of gut microbiome with the ICI treatment efficacy in patients with HCC has also been reported, there still remained enriched microbiome subclones in patients with disease control [37].

\section{Tumor heterogeneity challenges the effect of existed biomarkers in immunotherapy}

Till now, few biomarkers have been successfully translated into clinical implication of HCC. One possible reason for such biomarker directed immunotherapy is tumor heterogeneity. Tumor heterogeneity is the key feature of carcinogenesis and is linked with medication therapy failure [38, 39]. HCC is a typical tumor of extreme heterogeneity at genomic [40], epigenetic [41], transcriptional [42], and protein [43] level. Single-cell analysis also demonstrated that HCC cancer stem cells are composed of phenotypically, functionally, and transcriptomically heterogeneous subpopulations [44]. Even so, the tumor heterogeneity of HCC could still be classified into the following four categories: interpatient heterogeneity indicates differences between patients of the same tumor histology, intertumoral heterogeneity means differences between tumor foci in the same patient, intratumoral heterogeneity (ITH, spatial heterogeneity) is the differences among cells of the same tumor foci, and temporal heterogeneity describes the temporal changes in a tumor along treatment.

The four types of heterogeneity collectively contribute to medication therapy failure. Interpatient heterogeneity implies that a simple biomarker may not fit the whole patient population and personalized ICI treatment modality is necessary for better treatment response. However, the co-existence of intra- and inter-tumoral heterogeneity, with the latter one being much more profound and multifocal tumors being common in HCC, challenges precision immunotherapy since single tumor biopsy evaluation of PD-L1 expression or profiling genomic information could not represent the landscape of the whole tumor, let alone for multifocal HCCs. Thus, primary treatment resistance may happen. Even for tumors who are sensitive initially, the temporal heterogeneity, which is caused by selective pressure during treatment, would result in the expansion of rare subclones of resistant tumor cells and deem the eventual failure of agents. Indeed, single-cell transcriptomic analysis of liver tumors from patients treated with immunotherapy found an increase in tumor cell state heterogeneity was tightly linked to treatment response and patient prognosis [45].

\section{Liquid biopsy, the promising biomarker for cancer immunotherapy}

In the past years, liquid biopsy has emerged as an appealing source of new biomarkers and has been widely used in the management of cancer including HCC [46]. Accordingly, studies evaluating the predictors of benefit from ICI treatment have displayed a burgeoning interest in liquid biopsy. The analysts consist of liquid biopsy include circulating tumor DNA (ctDNA), circulating tumor cells (CTC), lymphocyte subpopulations, exosomes and metabolites to name a few. Liquid biopsy had several advantages over conventional single-site tissue sampling or serum protein markers, including higher 
sensitivity and specificity, non-nonvasiveness, dynamic monitoring, and the most important of all, overcoming the limit of spatial and temporal heterogeneity [47]. For HCC, tissue access is not always easily available and a single biopsy could hardly represent the whole genetic information, let alone in the case of multiple and huge HCCs. In the process of treatment (TACE, targeted therapy and immunotherapy), the selection pressure resulted clonal evolution surely leads to tumor genome change and drug resistance, and finally treatment failure. However, the genetic information from tissue biopsy prior to treatment does not contribute to treatment decision Meanwhile, repeated tumor biopsy is invasive and not operable in clinical practice. In such circumstance, liquid biopsy could be highly useful since venipuncture is nearly non-invasive and the genetic information from ctDNA and CTC has been demonstrated representative of the tumor genome [48]. Thus, with the application of liquid biopsy, it's able to reveal a much more comprehensive tumor genome and dynamically monitor the genetic change along treatment [49]. In general, liquid biopsy could help select candidate $\mathrm{HCC}$ patients who would benefit from immunotherapy in several aspects.

\section{Implementation of personalized immunotherapy}

Ideally, assessment of PD-L1 expression in tumor tissues could predict ICI response; however, there is a growing interest in assessment of PD-L1 mRNA and protein levels in circulating extracellular vesicles, which may have the potential to predict response to anti-PD-1/PD-L1 antibodies [50, 51]. For instance, CTCs from metastatic prostate cancer patients heterogeneously express immune checkpoints including B7H3, PD-L1, PD-L2, and CTLA-4, which suggests the detection of CTCs with these immune checkpoints may select patients suitable for immunotherapy [52]. In patients with metastatic genitourinary cancer received immunotherapy, higher CTC counts at baseline and presence of specific CTC morphologic subtypes, PD-L1 ${ }^{+}$CTCs, are associated with shorter survival [53]. Similarly, in head and neck squamous cell carcinoma patients, CTCs with PD-L1 overexpression are prognostic and the absence of PD-L1 overexpression in CTCs at the end of treatment strongly correlated with complete response [54]. Detection of PD-L1 positive CTC in peripheral blood of HCC patients is feasible and lays the foundation for real-time surveillance and individualized immunotherapy [55].

On the other hand, NGS based ctDNA detection in HCC patients may be of extreme usefulness for precision immunotherapy. ctDNA carries plenty of genetic information derived from tumor tissues and it could serve as supplement to tumor biopsy or solely provide profiling of tumor genome with high coverage [56].
Not only TMB but also genetic signatures and MSI/ dMMR could all be analyzed through ctDNA profiling. In the Phase I trial of small case series bearing solid tumors, TMB calculated from ctDNA correlated with that form tumor tissues and decrease in mutation variant allele frequency during treatment was observed in responders [57]. By retrospective analysis, Gandara et al. found ctDNA TMB reproducibly identified NSCLC (non-small cell lung cancer) patients who had obtained clinically significant improvements in PFS from atezolizumab as second-line treatment or higher [58]. Recently, commercial ctDNA platform for quantifying bTMB from plasma samples has been demonstrated to be feasible, accurate, and reproducible, with the optimal ctDNA TMB cutoff of $\geq 20$ mut/Mb predictive of clinical benefit with durvalumab + tremelimumab versus chemotherapy [59]. More importantly, if assayed with panels containing a greater number of mutations or larger panels, ctDNA could more accurately reflect tissue TMB [60, 61] and higher TMB in ctDNA was associated with longer PFS in NSCLC patients treated by ICIs [62].

Tumor mutations profiled through cfDNA sequencing is another effective biomarker to predict immunotherapy outcomes [63]. CTNNB1 gene mutations are reported to be predictive of response to immunotherapy in HCC patients $[64,65]$ but intratumoral heterogeneity and the presence of multifocal tumors challenge comprehensive molecular profiling from a single biopsy. Besides, mutational profiling of tumor biopsies taken prior to immunotherapy do not ensure continuous treatment response during the whole disease course. However, ctDNA could provide additional information of tumor mutations which were not apparent in single tumor biopsy and combining analysis of ctDNA and tumor tissue increased the detection rate of CTNNB1 mutation [66]. More commonly, ctDNA has been demonstrated quantifiable across all HCC stages and could specially detect mutations in the key driver genes [67].

NGS has been approved for detection of MSI/dMMR in patients with metastatic CRC before ICI treatment and such method was reported to perform at least as efficiently as the reference method [68]. Combination of NGS with liquid biopsy to identify cancer patients with $\mathrm{MSI}-\mathrm{H}$ is feasible in clinical practice and may overcome the limitation and invasiveness of tumor tissue biopsy [69]. A recent report has demonstrated NGS based liquid biopsy for MSI evaluation in clinical practice: 2 metastatic castration-resistant prostate cancer patients who failed multiple lines of therapy and were detected MSI-H in ctDNA assay had shown an excellent clinical response to pembrolizumab [70]. It's thus reasonable to propose NGS of ctDNA or single cell sequencing of CTC for MSI/dMMR analsysis in HCC. 


\section{Candidate selection for adjuvant immunotherapy}

The 5-year recurrence rate of HCC after hepatectomy could be as high as $70 \%$ and adjuvant treatments to prevent recurrence after curative liver resection is of unmet need [71]. Adjuvant immunotherapy has displayed survival benefit for patients undergone radical surgery in melanoma [72], esophageal or gastroesophageal junction cancer [73]. It's thus reasonable to propose such usage in $\mathrm{HCC}$, especially in patients with high recurrence risks. In fact, immunotherapy may play pivotal role in this kind of clinical setting [74]: adjuvant immunotherapy with activated autologous cytokine-induced killer cells increased recurrence-free and overall survival in patients who underwent curative treatment for HCC [75]. Although clinical trials evaluating the effect of adjuvant immunotherapy after hepatectomy are still ongoing (Table 2), it's not uncommon that surgical oncologist have already prescribed ICIs for patients at high risk of recurrence.

The underlying mechanism for indication of adjuvant immunotherapy may rest in minimal residual disease (MRD), which implies disseminated tumor cells (DTC) from the primary lesion to distant organs without any clinical or radiological evidence of metastasis at the time of initial treatment or residual tumor cells left behind after curative-intent surgery that eventually lead to local recurrence. The existence of MRD highly predicts tumor recurrence and metastasis. Moreover, DTCs could take advantage of the expression of checkpoint ligands such as PD-L1 to escape TIL antitumor activity, which means ICI-based anticancer strategy may be especially relevant against MRD. Thus, identification of HCC patients with MRD facilitates precise adjuvant immunotherapy whereas liquid biopsy has displayed extremely sensitivity for detection of MRD [76]. For example, in localized colon cancer, postoperative plasma ctDNA detected MRD and identified patients at high risk of relapse, preceding radiological recurrence with a median lead time of 11.5 months [77].

Actually, liquid biopsy guided adjuvant immunotherapy has already proved survival benefit in cancer patients. In the phase III IMvigor010 study, adjuvant atezolizumab did not prolong DFS in unselected patients with urothelial cancer who underwent surgery; however, in those who were tested positive for ctDNA, improvement in both DFS and overall survival was observed [78]. In comparison, no such differences were noted among trial participants who were negative for ctDNA in their blood in the overall study population [79].

In HCC, microvascular invasion (MVI) implies disseminated tumor cells in the adjacent liver tissues and is a common type of MRD. Interestingly, positive ctDNA status after liver resection correlated with MVI and predicted early tumor recurrence of HCC [80]. Similarly, CTC loads decreased immediately after hepatectomy in HCC patients and those with persistently high postoperative CTC load had a high probability of tumor recurrence [81]. Moreover, patients with postoperative CTC load $\geq 3$ bear higher risk of extrahepatic metastasis after curative surgical resection of HCC and they should be recommended with close surveillance program to timely implement interventions [82]. In fact, postoperative CTC count was found to be an effective indicator for the administration of adjuvant TACE (transcatheter arterial chemoembolization) since adjuvant TACE could reduce early recurrence in HCC patients with positive CTC status after curative-intent hepatectomy [83]. Thus, detection postoperative ctDNA status or CTC load may be a viable option for $\mathrm{HCC}$ patients after surgery and positive ctDNA expression or CTC load could be an indication for adjuvant immunotherapy. More commonly, identifying MRD-positive HCC patients will surely provide additional benefit, regardless of the type of adjuvant ICI treatment, or even other modalities.

Table 2 Postoperative adjuvant immunotherapy trials for HCC

\begin{tabular}{|c|c|c|c|}
\hline Identifier No. & Interventions & Setting & $\begin{array}{l}\text { Primary } \\
\text { outcome }\end{array}$ \\
\hline $\begin{array}{l}\text { NCT03859128 (JUPITER } \\
\text { 04) }\end{array}$ & Toripalimab or placebo & Phase 2, 3 & RFS \\
\hline $\begin{array}{l}\text { NCT03383458 } \\
\text { (CheckMate 9DX) }\end{array}$ & Nivolumab or placebo & Phase 3 & RFS \\
\hline $\begin{array}{l}\text { NCT03867084 (KEYNOTE- } \\
\text { 937) }\end{array}$ & Pembrolizumab & Phase 3, Double-blinded, Placebo controled & RFS, OS \\
\hline NCT04639180 & Camrelizumab plus Apatinib & & \\
\hline $\begin{array}{l}\text { NCT04102098 } \\
\text { (IMbrave050) }\end{array}$ & Atezolizumab plus Bevacizumab & $\begin{array}{l}\text { Phase } 3 \text {, open label, two arms (versus active } \\
\text { surveillence) }\end{array}$ & RFS \\
\hline $\begin{array}{l}\text { NCT03847428 (EMERALD- } \\
\text { 2) }\end{array}$ & $\begin{array}{l}\text { Durvalumab plus bevacizumab or durvalumab } \\
\text { monotherapy or placebo }\end{array}$ & $\begin{array}{l}\text { Phase 3, randomized, double-blind, placebo- } \\
\text { controlled }\end{array}$ & RFS \\
\hline NCT04418401 (CISLD-8) & Donafenib plus any anti-PD-1 antibody & Phase 1 & 1-year RFS \\
\hline NCT04233840 & Nivolumab and Ropeginterferon alfa-2b & Phase I/II Open Label & RFS \\
\hline
\end{tabular}




\section{Dynamic treatment surveillance}

Apart from being as an indicator of adjuvant immunotherapy, liquid biopsy could also dynamically, and is especially suitable for, monitor ICI treatment response [84-87] (Table 3). Traditionally, treatment response is mainly judged by comparison of tumor size on imaging. But this kind of assessment bears the disadvantages of low sensitivity, radioactivity, and most of all, not timely enough. Besides, the treatment effects of immunotherapy are challenging to interpret since tumors often shrink slowly or become enlarged due to inflammation (radiological pseudoprogression). What's more important, the selective pressure during immunotherapy could induce clonal evolution, which mean the tumor genome differs from that profiled before treatment. Unfortunately, conventional techniques are almost impossible to dynamically monitor such changes via longitudinal and real-time analysis.

Liquid biopsy, however, has the potential of determining tumor evolution patterns and therapeutic responses in a dynamic and non-invasive manner. In lung cancer and urothelial cancer, change in variant allele frequency of somatic mutations in ctDNA strongly correlated with ICIs treatment duration, activity, and outcomes [88]. Both CTC numbers and PD-L1 expression on CTCs potentially indicate disease progression during the disease course of urothelial carcinoma patients receiving ICIs [89]. Decreased ctDNA level served as an early marker of therapeutic efficacy and predicted significantly prolonged survival in lung cancer patients treated with ICIs [90]. Longitudinal analysis of ctDNA with custom NGS gene panel allowed for disease burden monitoring during immunotherapy in melanoma patients [91] and a persistently elevated ctDNA during treatment indicated poor prognosis and the necessity of combination and sequencing of subsequent therapies [92]. Moreover, undetectable ctDNA at baseline or detectable ctDNA at baseline followed by $>10$-fold decrease during immunotherapy was associated with pseudoprogression while patients experienced true progression had detectable ctDNA at baseline which remained stable or increased [93]. These experience could be readily used for reference in HCC. In fact, ctDNA profiling during lenvatinib treatment was a useful marker of disease progression [94] and serial profiling of ctDNA using targeted ultra-deep sequencing correlated with systemic treatment response [95].

\section{Unraveling immunotherapy resistance mechanism}

Nonetheless, the response to ICIs in HCC is $20-30 \%$ and patients would invariably undergo disease progression. Though other therapeutics could still be used following ICI exposure, it's necessary to explore the mechanisms of ICI resistance and the combination of TKIs with ICI or as subsequent therapy. As mentioned above, tumor heterogeneity is the key factor contributing to immunotherapy resistance, either primary or acquired. Thus, techniques which could circumvent the obstacles brought by tumor heterogeneity can surely help unveil the underlying mechanisms and evidence from other malignancies highlights the importance of liquid biopsy in these aspects. By liquid biopsy monitoring of tumor subclones over treatment, Stein at al found ctDNA showed rapid clearance in the majority of patients mirroring a high rate of early tumor shrinkage and loss of PD-L1 on the tumor cell surface by PD-L1 mutations abrogated direct antitumor effects of PD-L1 antibody, avelumab [96]. Moreover, such mutations were present already months before clinical resistance occurred, which supported the notion that temporal heterogeneity caused by selective pressure resulted in acquired resistance. Detection of PD-L1+ aneuploid CTCs and circulating tumor endothelial cells in histopathologic PD-L1 negative NSCLC patients indicated that ITH resulted in primary resistance to anti-PD-1 treatment [97].

Liquid biopsy could also discriminate immunotherapy resistance from pseudo-progression. Pseudo-progression happens in the early stage of immunotherapy when larger tumor lesions or new lesions occur as a result of the inflammatory reaction induced by ICI, before the tumor actually shrinks. Generally, it's not easy to distinguish pseudo-progression from immunotherapy resistance

Table 3 Clinical trials evaluating biomarkers of response in HCC patients treated by immunotherapy

\begin{tabular}{|c|c|c|c|}
\hline Identifier No. & Biomarker type & Treatment & Patients \\
\hline NCT05044676 & Circulating Immune Cells & Atezolizumab/Bevacizumab & Advanced HCC \\
\hline $\begin{array}{l}\text { NCT04965454 (EXTRACT- } \\
\text { HCC) }\end{array}$ & Genomic liquid biopsy based biomarkers & $\mathrm{ICls}$ & Inoperable HCC \\
\hline NCT03895970 & Not specified & $\begin{array}{l}\text { Lenvatinib plus } \\
\text { Pembrolizumab }\end{array}$ & Advanced hepatobiliary tumors \\
\hline NCT04642664 (ACABC) & Not specified & Apatinib plus Camrelizumab & Hepatobiliary Neoplasm \\
\hline $\begin{array}{l}\text { NCT03595813 (IMMUNO- } \\
\text { SUP) }\end{array}$ & Plasma immunosuppressive actors & $\mathrm{ICls}$ & $\begin{array}{l}\text { Locally advanced or metastatic solid } \\
\text { tumor }\end{array}$ \\
\hline NCT03514368 (MINER) & $\begin{array}{l}\text { Immunological biomarkers from blood } \\
\text { samples }\end{array}$ & $\mathrm{ICls}$, alone or in combination & Any advanced solid tumor \\
\hline
\end{tabular}


using imaging modalities. However, the two different biological process could be effectively judged by liquid biopsy methods since in cases with pseudo-progression, the ctDNA levels decrease rapidly and persistently, whereas treatment resistance associated disease progression usually have significant increases in ctDNA amount. Indeed, Takai et al. reported a melanoma patient experienced immediate decrease of ctDNA content surge after a surge at the time of pseudo-progression [98].

\section{Conclusions}

The advent of ICIs has greatly changed the management and outcome of $\mathrm{HCC}$ while biomarkers predicting ICI treatment efficacy are still of unmet need. Future studies are urgently needed to evaluate the value of utilizing liquid biopsy analytes for therapeutic decision making by identification of HCC patients who would benefit from or are nonresponders to ICI treatment. The findings on the liquid biopsy would undoubtfully reveal the fundamental role of such analytes in HCC immunotherapy, with foreseeable and not-far-to-come clinical applications.

\begin{abstract}
Abbreviations
HCC: hepatocellular carcinoma; ICl: immune checkpoint inhibitor; CtDNA: circulating tumor DNA; CTC: circulating tumor cell; PD-

1: programmed cell death-1; PD-L1: programmed cell death-1ligand; CTLA4: cytotoxic T-lymphocyte-associated antigen-4; irAE: immune-related adverse event; OS: overall survival; DFS: disease-free survival; TMB: tumor mutation burden; HPD: hyperprogressive disease; ITH: intratumoral heterogeneity; dMMR/MSI: deficient in mismatch repair /microsatellite instability; LT: liver transplantation; TME: tumor microenvironment; TACE: transcatheter arterial chemoembolization; NASH: non-alcoholic steatohepatitis; MVI: microvascular invasion; MRD: minimal residual disease; DTC: disseminated tumor cell; NGS: next generation sequencing; NSCLC: non-small cell lung cancer
\end{abstract}

\section{Acknowledgements}

None.

\section{Authors' contributions}

Huang Ao and Zhang Xin made literature review and conducted draft writing. Zhou Jian proposed the concept and revised the manuscript. All authors read and approved the final manuscript.

\section{Funding}

This study was jointly supported by the National Key R\&D Program of China (2019YFC1315800, 2019YFC1315802), National Natural Science Foundation of China (No.82150004, 81830102, 81772578, 81802991), Fudan University (IDF152064/014), Zhongshan Hospital Fudan University (2021ZSYQ09), and Shanghai Municipal Key Clinical Specialty.

\section{Availability of data and materials}

Not applicable.

\section{Declarations}

Ethical approval and consent to participate

Not applicable.

\section{Consent for publication}

Not applicable.

\section{Competing interests}

The authors declare that they have no conflict of interest.

\section{Author details}

${ }^{1}$ Department of Liver Surgery and Transplantation, Zhongshan Hospital, Fudan University; Key Laboratory of Carcinogenesis and Cancer Invasion (Fudan University), Ministry of Education; Shanghai Key Laboratory of Organ Transplantation, Zhongshan Hospital, Fudan University, Shanghai 200032, China. 'Institute of Biomedical Sciences, Fudan University, Shanghai 200032, China. ${ }^{3}$ State Key Laboratory of Genetic Engineering, Fudan University, Shanghai 200032, China. ${ }^{4}$ Liver Cancer Institute, Zhongshan Hospital, Fudan University, 136 Yi Xue Yuan Road, Shanghai 200032, China.

Received: 29 September 2021 Accepted: 3 November 2021

Published online: 20 December 2021

\section{References}

1. Sangro B, Sarobe P, Hervás-Stubbs S, Melero I. Advances in immunotherapy for hepatocellular carcinoma. Nat Rev. Gastroenterol Hepatol. 2021;18: 525-43.

2. Yau T, Kang YK, Kim TY, El-Khoueiry AB, Santoro A, Sangro B, et al. Efficacy and Safety of Nivolumab Plus Ipilimumab in Patients With Advanced Hepatocellular Carcinoma Previously Treated With Sorafenib: The CheckMate 040 Randomized Clinical Trial. JAMA Oncol. 2020;6:e204564.

3. Dyhl-Polk A, Mikkelsen MK, Ladekarl M, Nielsen DL. Clinical Trials of Immune Checkpoint Inhibitors in Hepatocellular Carcinoma. J Clin Med. 2021;10.

4. Gao Q, Wang XY, Qiu SJ, Yamato I, Sho M, Nakajima Y, et al. Overexpression of PD-L1 significantly associates with tumor aggressiveness and postoperative recurrence in human hepatocellular carcinoma. Clin Cancer Res. 2009;15:971-9.

5. Calderaro J, Rousseau B, Amaddeo G, Mercey M, Charpy C, Costentin C, et al. Programmed death ligand 1 expression in hepatocellular carcinoma: Relationship With clinical and pathological features. Hepatology. 2016;64: 2038-46.

6. Jung HI, Jeong D, Ji S, Ahn TS, Bae SH, Chin S, et al. Overexpression of PDL1 and PD-L2 Is Associated with Poor Prognosis in Patients with Hepatocellular Carcinoma. Cancer Res Treat. 2017;49:246-54.

7. Umemoto Y, Okano S, Matsumoto Y, Nakagawara H, Matono R, Yoshiya S, et al. Prognostic impact of programmed cell death 1 ligand 1 expression in human leukocyte antigen class I-positive hepatocellular carcinoma after curative hepatectomy. J Gastroenterol. 2015;50:65-75.

8. Dai X, Xue J, Hu J, Yang SL, Chen GG, Lai PBS, et al. Positive Expression of Programmed Death Ligand 1 in Peritumoral Liver Tissue is Associated with Poor Survival after Curative Resection of Hepatocellular Carcinoma. Transl Oncol. 2017:10:511-7.

9. Sangro B, Melero I, Wadhawan S, Finn RS, Abou-Alfa GK, Cheng AL, et al. Association of inflammatory biomarkers with clinical outcomes in nivolumab-treated patients with advanced hepatocellular carcinoma. J Hepatol. 2020;73:1460-9.

10. Finn RS, Ryoo BY, Merle P, Kudo M, Bouattour M, Lim HY, et al. Pembrolizumab As Second-Line Therapy in Patients With Advanced Hepatocellular Carcinoma in KEYNOTE-240: A Randomized, Double-Blind. Phase III Trial. J Clin Oncol. 2020;38:193-202.

11. Li JH, Ma WJ, Wang GG, Jiang X, Chen X, Wu L, et al. Clinicopathologic Significance and Prognostic Value of Programmed Cell Death Ligand 1 (PDL1) in Patients With Hepatocellular Carcinoma: A Meta-Analysis. Front Immunol. 2018;9:2077.

12. Shi GM, Wang J, Huang XW, Huang XY, He YF, Ji Y, et al. Graft Programmed Death Ligand 1 Expression as a Marker for Transplant Rejection Following Anti-Programmed Death 1 Immunotherapy for Recurrent Liver Tumors. Liver Transpl. 2021:27:444-9

13. Amjad W, Kotiah S, Gupta A, Morris M, Liu L, Thuluvath PJ. Successful Treatment of Disseminated Hepatocellular Carcinoma After Liver Transplantation With Nivolumab. J Clin Exp Hepatol. 2020;10:185-7.

14. Qiu J, Tang W, Du C. Immune Checkpoint Inhibitors in Patients with Recurrent Hepatocellular Carcinoma after Liver Transplantation: A Case Report and Literature Review. Curr Cancer Drug Targets. 2020;20:720-7.

15. Yarchoan M, Hopkins A, Jaffee EM. Tumor Mutational Burden and Response Rate to PD-1 Inhibition. N Engl J Med. 2017;377:2500-1.

16. Forde PM, Chaft JE, Smith KN, Anagnostou V, Cottrell TR, Hellmann MD, et al. Neoadjuvant PD-1 Blockade in Resectable Lung Cancer. N Engl J Med. 2018;378:1976-86

17. Li L, Rao X, Wen Z, Ding X, Wang X, Xu W, et al. Implications of driver genes associated with a high tumor mutation burden identified using next- 
generation sequencing on immunotherapy in hepatocellular carcinoma. Oncol Lett. 2020;19:2739-48.

18. Aguiar PN Jr, Tadokoro H, Forones NM, de Mello RA. MMR deficiency may lead to a high immunogenicity and then an improvement in anti-PD-1 efficacy for metastatic colorectal cancer. Immunotherapy. 2015;7:1133-4.

19. Le DT, Uram JN, Wang H, Bartlett BR, Kemberling H, Eyring AD, et al. PD-1 Blockade in Tumors with Mismatch-Repair Deficiency. N Engl J Med. 2015; 372:2509-20.

20. Cohen R, Hain E, Buhard O, Guilloux A, Bardier A, Kaci R, et al. Association of Primary Resistance to Immune Checkpoint Inhibitors in Metastatic Colorectal Cancer With Misdiagnosis of Microsatellite Instability or Mismatch Repair Deficiency Status. JAMA Oncol. 2019;5:551-5.

21. Pietrantonio F, Miceli R, Raimondi A, Kim YW, Kang WK, Langley RE, et al. Individual Patient Data Meta-Analysis of the Value of Microsatellite Instability As a Biomarker in Gastric Cancer. J Clin Oncol. 2019;37:3392-400.

22. Pietrantonio F, Randon G, Di Bartolomeo M, Luciani A, Chao J, Smyth EC, et al. Predictive role of microsatellite instability for PD-1 blockade in patients with advanced gastric cancer: a meta-analysis of randomized clinical trials. ESMO Open. 2021;6:100036.

23. Pietrantonio F, Loupakis F, Randon G, Raimondi A, Salati M, Trapani D, et al. Efficacy and Safety of Immune Checkpoint Inhibitors in Patients with Microsatellite Instability-High End-Stage Cancers and Poor Performance Status Related to High Disease Burden. Oncologist. 2020;25:803-9.

24. Le DT, Durham JN, Smith KN, Wang H, Bartlett BR, Aulakh LK, et al. Mismatch repair deficiency predicts response of solid tumors to PD-1 blockade. Science. 2017:357:409-13.

25. Morita M, Nishida N, Sakai K, Aoki T, Chishina H, Takita M, et al. Immunological Microenvironment Predicts the Survival of the Patients with Hepatocellular Carcinoma Treated with Anti-PD-1 Antibody. Liver Cancer. 2021;10:380-93.

26. Cao D, Chen MK, Zhang QF, Zhou YF, Zhang MY, Mai SJ, et al. Identification of immunological subtypes of hepatocellular carcinoma with expression profiling of immune-modulating genes. Aging (Albany NY). 2020;12:12187-205.

27. Kim HD, Song GW, Park S, Jung MK, Kim MH, Kang HJ, et al. Association Between Expression Level of PD1 by Tumor-Infiltrating CD8(+) T Cells and Features of Hepatocellular Carcinoma. Gastroenterology. 2018;155:1936-50. e17.

28. Pfister D, Núñez NG, Pinyol R, Govaere O, Pinter M, Szydlowska M, et al. NASH limits anti-tumour surveillance in immunotherapy-treated HCC. Nature. 2021;592:450-6.

29. Guven DC, Aktas BY, Simsek C, Aksoy S. Gut microbiota and cancer immunotherapy: prognostic and therapeutic implications. Future Oncol. 2020;16:497-506.

30. Gharaibeh RZ, Jobin C. Microbiota and cancer immunotherapy: in search of microbial signals. Gut. 2019;68:385-8.

31. Yu LX, Schwabe RF. The gut microbiome and liver cancer: mechanisms and clinical translation. Nat Rev. Gastroenterol Hepatol. 2017;14:527-39.

32. Schwabe RF, Greten TF. Gut microbiome in HCC - Mechanisms, diagnosis and therapy. J Hepatol. 2020;72:230-8.

33. Ponziani FR, Bhoori S, Castelli C, Putignani L, Rivoltini L, Del Chierico F, et al. Hepatocellular Carcinoma Is Associated With Gut Microbiota Profile and Inflammation in Nonalcoholic Fatty Liver Disease. Hepatology. 2019;69: 107-20.

34. Behary J, Amorim N, Jiang XT, Raposo A, Gong L, McGovern E, et al. Gut microbiota impact on the peripheral immune response in non-alcoholic fatty liver disease related hepatocellular carcinoma. Nat Commun. 2021;12: 187.

35. Li L, Ye J. Characterization of gut microbiota in patients with primary hepatocellular carcinoma received immune checkpoint inhibitors: A Chinese population-based study. Medicine (Baltimore). 2020;99:e21788.

36. Zheng $Y$, Wang T, Tu X, Huang Y, Zhang H, Tan D, et al. Gut microbiome affects the response to anti-PD-1 immunotherapy in patients with hepatocellular carcinoma. J Immunother Cancer. 2019;7:193.

37. Shen YC, Lee PC, Kuo YL, Wu WK, Chen CC, Lei CH, et al. An Exploratory Study for the Association of Gut Microbiome with Efficacy of Immune Checkpoint Inhibitor in Patients with Hepatocellular Carcinoma. J Hepatocell Carcinoma. 2021;8:809-22.

38. Gillies RJ, Verduzco D, Gatenby RA. Evolutionary dynamics of carcinogenesis and why targeted therapy does not work. Nat Rev. Cancer. 2012;12:487-93.

39. Fisher R, Pusztai L, Swanton C. Cancer heterogeneity: implications for targeted therapeutics. Br J Cancer. 2013;108:479-85.
40. Ling S, Hu Z, Yang Z, Yang F, Li Y, Lin P, et al. Extremely high genetic diversity in a single tumor points to prevalence of non-Darwinian cell evolution. Proc Natl Acad Sci U S A. 2015;112:E6496-505.

41. Ding X, He M, Chan AWH, Song QX, Sze SC, Chen H, et al. Genomic and Epigenomic Features of Primary and Recurrent Hepatocellular Carcinomas. Gastroenterology. 2019;157:1630-45.e6.

42. Xu LX, He MH, Dai ZH, Yu J, Wang JG, Li XC, et al. Genomic and transcriptional heterogeneity of multifocal hepatocellu ar carcinoma. Ann Oncol. 2019.

43. Friemel J, Rechsteiner M, Frick L, Bohm F, Struckmann K, Egger M, et al. Intratumor heterogeneity in hepatocellular carcinoma. Clin Cancer Res. 2015;21:1951-61.

44. Z Zheng H, Pomyen Y, Hernandez MO, Li C, Livak F, Tang W, et al. Single-cell analysis reveals cancer stem cell heterogeneity in hepatocellular carcinoma. Hepatology. 2018:68:127-40.

45. Ma L, Wang L, Khatib SA, Chang CW, Heinrich S, Dominguez DA, et al. Single-cell atlas of tumor cell evolution in response to therapy in hepatocellular carcinoma and intrahepatic cholangiocarcinoma. J Hepatol. 2021.

46. Zhou J, Huang A, Yang XR. Liquid Biopsy and its Potential for Management of Hepatocellular Carcinoma. J Gastrointest Cancer. 2016:47:157-67.

47. Siravegna G, Marsoni S, Siena S, Bardelli A. Integrating liquid biopsies into the management of cancer. Nat Rev. Clin Oncol. 2017;14:531-48.

48. Mohanty A, Mohanty SK, Rout S, Pani C. Liquid Biopsy, the hype vs. hope in molecular and clinical oncology. Semin Oncol. 2021.

49. Tran NH, Kisiel J, Roberts LR. Using cell-free DNA for HCC surveillance and prognosis. JHEP Rep. 2021;3:100304.

50. Del Re M, van Schaik RHN, Fogli S, Mathijssen RHJ, Cucchiara F, Capuano A, et al. Blood-based PD-L1 analysis in tumor-derived extracellular vesicles: Applications for optimal use of anti-PD-1/PD-L1 axis inhibitors. Biochim Biophys Acta Rev. Cancer. 2021;1875:188463.

51. Wang J, Zeng H, Zhang H, Han Y. The role of exosomal PD-L1 in tumor immunotherapy. Transl Oncol. 2021;14:101047.

52. Zhang T, Agarwal A, Almquist RG, Runyambo D, Park S, Bronson E, et al. Expression of immune checkpoints on circulating tumor cells in men with metastatic prostate cancer. Biomark Res. 2021;9:14.

53. Chalfin HJ, Pramparo T, Mortazavi A, Niglio SA, Schonhoft JD, Jendrisak A, et al. Circulating Tumor Cell Subtypes and T-cell Populations as Prognostic Biomarkers to Combination Immunotherapy in Patients with Metastatic Genitourinary Cancer. Clin Cancer Res. 2021;27:1391-8.

54. Strati A, Koutsodontis G, Papaxoinis G, Angelidis I, Zavridou M, Economopoulou P, et al. Prognostic significance of PD-L1 expression on circulating tumor cells in patients with head and neck squamous cell carcinoma. Ann Oncol. 2017;28:1923-33.

55. Yue C, Jiang Y, Li P, Wang Y, Xue J, Li N, et al. Dynamic change of PD-L1 expression on circulating tumor cells in advanced solid tumor patients undergoing PD-1 blockade therapy. Oncoimmunology. 2018;7:e1438111.

56. Ng CKY, Di Costanzo GG, Tosti N, Paradiso V, Coto-Llerena M, Roscigno G, et al. Genetic profiling using plasma-derived cell-free DNA in therapy-naïve hepatocellular carcinoma patients: a pilot study. Ann Oncol. 2018;29:128691.

57. Araujo DV, Wang A, Torti D, Leon A, Marsh K, McCarthy A, et al. Applications of Circulating Tumor DNA in a Cohort of Phase I Solid Tumor Patients Treated With Immunotherapy. JNCI Cancer Spectr. 2021;5:pkaa122.

58. Gandara DR, Paul SM, Kowanetz M, Schleifman E, Zou W, Li Y, et al. Bloodbased tumor mutational burden as a predictor of clinical benefit in nonsmall-cell lung cancer patients treated with atezolizumab. Nat Med. 2018;24: 1441-8.

59. Si H, Kuziora M, Quinn KJ, Helman E, Ye J, Liu F, et al. A Blood-based Assay for Assessment of Tumor Mutational Burden in First-line Metastatic NSCLC Treatment: Results from the MYSTIC Study. Clin Cancer Res. 2021:27:1631-40.

60. Chae YK, Davis AA, Agte S, Pan A, Simon NI, lams WT, et al. Clinical Implications of Circulating Tumor DNA Tumor Mutational Burden (ctDNA TMB) in Non-Small Cell Lung Cancer. Oncologist. 2019;24:820-8.

61. Wang Z, Duan J, Cai S, Han M, Dong H, Zhao J, et al. Assessment of Blood Tumor Mutational Burden as a Potential Biomarker for Immunotherapy in Patients With Non-Small Cell Lung Cancer With Use of a Next-Generation Sequencing Cancer Gene Panel. JAMA Oncol. 2019;5:696-702.

62. Chen $X$, Fang $L$, Zhu $Y$, Bao Z, Wang $Q$ Liu $R$, et al. Blood tumor mutation burden can predict the clinical response to immune checkpoint inhibitors 
in advanced non-small cell lung cancer patients. Cancer Immuno Immunother. 2021.

63. Li S, Noor ZS, Zeng W, Stackpole ML, Ni X, Zhou Y, et al. Sensitive detection of tumor mutations from blood and its application to immunotherapy prognosis. Nat Commun. 2021;12:4172.

64. Harding JJ, Nandakumar S, Armenia J, Khalil DN, Albano M, Ly M, et al. Prospective Genotyping of Hepatocellular Carcinoma: Clinical Implications of Next-Generation Sequencing for Matching Patients to Targeted and Immune Therapies. Clin Cancer Res. 2019;25:2116-26.

65. Ruiz de Galarreta M, Bresnahan E, Molina-Sánchez P, Lindblad KE, Maier B, Sia D, et al. $\beta$-Catenin Activation Promotes Immune Escape and Resistance to Anti-PD-1 Therapy in Hepatocellular Carcinoma. Cancer Discov. 2019;9: $1124-41$.

66. Oversoe SK, Clement MS, Weber B, Grønbæk H, Hamilton-Dutoit SJ, Sorensen BS, et al. Combining tissue and circulating tumor DNA increases the detection rate of a CTNNB1 mutation in hepatocellular carcinoma. BMC Cancer. 2021:21:376

67. Howell J, Atkinson SR, Pinato DJ, Knapp S, Ward C, Minisini R, et al. Identification of mutations in circulating cell-free tumour DNA as a biomarker in hepatocellular carcinoma. Eur J Cancer. 2019;116:56-66.

68. Ratovomanana $T$, Cohen $R$, Svrcek M, Renaud F, Cervera P, Siret A, et al. Performance of Next-Generation Sequencing for the Detection of Microsatellite Instability in Colorectal Cancer With Deficient DNA Mismatch Repair. Gastroenterology. 2021;161:814-26.e7.

69. Barata P, Agarwal N, Nussenzveig R, Gerendash B, Jaeger E, Hatton W, et al. Clinical activity of pembrolizumab in metastatic prostate cancer with microsatellite instability high (MSI-H) detected by circulating tumor DNA. J Immunother Cancer. 2020;8.

70. Ravindranathan D, Russler GA, Yantorni L, Drusbosky LM, Bilen MA. Detection of Microsatellite Instability via Circulating Tumor DNA and Response to Immunotherapy in Metastatic Castration-Resistant Prostate Cancer: A Case Series. Case Rep Oncol. 2021;14:190-6.

71. Zhang W, Zhang B, Chen XP. Adjuvant treatment strategy after curative resection for hepatocellular carcinoma. Front Med. 2021;15:155-69.

72. Eggermont AM, Chiarion-Sileni V, Grob JJ, Dummer R, Wolchok JD, Schmidt $\mathrm{H}$, et al. Adjuvant ipilimumab versus placebo after complete resection of high-risk stage III melanoma (EORTC 18071): a randomised, double-blind, phase 3 trial. Lancet Oncol. 2015;16:522-30.

73. Kelly RJ, Ajani JA, Kuzdzal J, Zander T, Van Cutsem E, Piessen G, et al. Adjuvant Nivolumab in Resected Esophageal or Gastroesophageal Junction Cancer. N Engl J Med. 2021;384:1191-203.

74. Brown ZJ, Greten TF, Heinrich B. Adjuvant Treatment of Hepatocellular Carcinoma: Prospect of Immunotherapy. Hepatology. 2019;70:1437-42.

75. Lee JH, Lee JH, Lim YS, Yeon JE, Song TJ, Yu SJ, et al. Adjuvant immunotherapy with autologous cytokine-induced killer cells for hepatocellular carcinoma. Gastroenterology. 2015;148:1383-91.e6.

76. Pantel K, Alix-Panabières C. Liquid biopsy and minimal residual disease latest advances and implications for cure. Nat Rev. Clin Oncol. 2019;16:40924.

77. Tarazona N, Gimeno-Valiente F, Gambardella V, Zuñiga S, Rentero-Garrido P, Huerta $M$, et al. Targeted next-generation sequencing of circulating-tumor DNA for tracking minimal residual disease in localized colon cancer. Ann Oncol. 2019;30:1804-12.

78. Powles T, Assaf ZJ, Davarpanah N, Banchereau R, Szabados BE, Yuen KC, et al. ctDNA guiding adjuvant immunotherapy in urothelial carcinoma. Nature. 2021.

79. Bellmunt J, Hussain M, Gschwend JE, Albers P, Oudard S, Castellano D, et al. Adjuvant atezolizumab versus observation in muscle-invasive urothelial carcinoma (IMvigor010): a multicentre, open-label, randomised, phase 3 trial. Lancet Oncol. 2021;22:525-37.

80. Wang J, Huang A, Wang YP, Yin Y, Fu PY, Zhang X, et al. Circulating tumor DNA correlates with microvascular invasion and predicts tumor recurrence of hepatocellular carcinoma. Ann Transl Med. 2020;8:237.

81. Guo W, Sun YF, Shen MN, Ma XL, Wu J, Zhang CY, et al. Circulating Tumor Cells with Stem-Like Phenotypes for Diagnosis, Prognosis, and Therapeutic Response Evaluation in Hepatocellular Carcinoma. Clin Cancer Res. 2018;24: 2203-13.

82. Sun YF, Wang PX, Cheng JW, Gong ZJ, Huang A, Zhou KQ, et al. Postoperative circulating tumor cells: An early predictor of extrahepatic metastases in patients with hepatocellular carcinoma undergoing curative surgical resection. Cancer Cytopathol. 2020;128:733-45.
83. Wang PX, Sun YF, Zhou KQ, Cheng JW, Hu B, Guo W, et al. Circulating tumor cells are an indicator for the administration of adjuvant transarterial chemoembolization in hepatocellular carcinoma: A single-center, retrospective, propensity-matched study. Clin Transl Med. 2020;10:e137.

84. Abbosh C, Birkbak NJ, Swanton C. Early stage NSCLC - challenges to implementing ctDNA-based screening and MRD detection. Nat Rev. Clin Oncol. 2018;15:577-86

85. Tie J, Cohen JD, Wang Y, Christie M, Simons K, Lee M, et al. Circulating Tumor DNA Analyses as Markers of Recurrence Risk and Benefit of Adjuvant Therapy for Stage III Colon Cancer. JAMA Oncol. 2019;5:1710-7.

86. Tie J, Cohen JD, Wang Y, Li L, Christie M, Simons K, et al. Serial circulating tumour DNA analysis during multimodality treatment of locally advanced rectal cancer: a prospective biomarker study. Gut. 2019;68:663-71.

87. Lee B, Lipton L, Cohen J, Tie J, Javed AA, Li L, et al. Circulating tumor DNA as a potential marker of adjuvant chemotherapy benefit following surgery for localized pancreatic cancer. Ann Oncol. 2019;30:1472-8.

88. Raja R, Kuziora M, Brohawn PZ, Higgs BW, Gupta A, Dennis PA, et al. Early Reduction in ctDNA Predicts Survival in Patients with Lung and Bladder Cancer Treated with Durvalumab. Clin Cancer Res. 2018;24:6212-22.

89. Bergmann S, Coym A, Ott L, Soave A, Rink M, Janning M, et al. Evaluation of PD-L1 expression on circulating tumor cells (CTCS) in patients with advanced urothelial carcinoma (UC). Oncoimmunology. 2020;9:1738798.

90. Goldberg SB, Narayan A, Kole AJ, Decker RH, Teysir J, Carriero NJ, et al. Early Assessment of Lung Cancer Immunotherapy Response via Circulating Tumor DNA. Clin Cancer Res. 2018;24:1872-80.

91. Berger M, Thueringer A, Franz D, Dandachi N, Talakić E, Richtig G, et al. Circulating Tumor DNA as a Marker for Treatment Response in Metastatic Melanoma Patients Using Next-Generation Sequencing-A Prospective Feasibility Study. Cancers (Basel). 2021;13.

92. Lee JH, Long GV, Boyd S, Lo S, Menzies AM, Tembe V, et al. Circulating tumour DNA predicts response to anti-PD1 antibodies in metastatic melanoma. Ann Oncol. 2017;28:1130-6.

93. Lee JH, Long GV, Menzies AM, Lo S, Guminski A, Whitbourne K, et al. Association Between Circulating Tumor DNA and Pseudoprogression in Patients With Metastatic Melanoma Treated With Anti-Programmed Cell Death 1 Antibodies. JAMA Oncol. 2018;4:717-21.

94. Fujii Y, Ono A, Hayes CN, Aikata H, Yamauchi M, Uchikawa S, et al. Identification and monitoring of mutations in circulating cell-free tumor DNA in hepatocellular carcinoma treated with lenvatinib. J Exp Clin Cancer Res. 2021;40:215

95. von Felden J, Craig AJ, Garcia-Lezana T, Labgaa I, Haber PK, D’Avola D, et al. Mutations in circulating tumor DNA predict primary resistance to systemic therapies in advanced hepatocellular carcinoma. Oncogene. 2021:40:140-51.

96. Stein A, Simnica D, Schultheiß C, Scholz R, Tintelnot J, Gökkurt E, et al. PDL1 targeting and subclonal immune escape mediated by PD-L1 mutations in metastatic colorectal cancer. J Immunother Cancer. 2021;9.

97. Zhang L, Zhang X, Liu Y, Zhang T, Wang Z, Gu M, et al. PD-L1(+) aneuploid circulating tumor endothelial cells (CTECS) exhibit resistance to the checkpoint blockade immunotherapy in advanced NSCLC patients. Cancer Lett. 2020;469:355-66.

98. Takai E, Omata W, Totoki Y, Nakamura H, Shiba S, Takahashi A, et al. Clonal dynamics of circulating tumor DNA during immune checkpoint blockade therapy for melanoma. Cancer Sci. 2021.

\section{Publisher's Note}

Springer Nature remains neutral with regard to jurisdictional claims in published maps and institutional affiliations.

\section{Ready to submit your research? Choose BMC and benefit from}

- fast, convenient online submission

- thorough peer review by experienced researchers in your field

- rapid publication on acceptance

- support for research data, including large and complex data types

- gold Open Access which fosters wider collaboration and increased citations

- maximum visibility for your research: over $100 \mathrm{M}$ website views per year

At $\mathrm{BMC}$, research is always in progress.

Learn more biomedcentral.com/submission 